\title{
Depression indicators in a national sample of older community and care home patients: applying the Quality and Outcomes Framework
}

\author{
Tess Harris, Sunil M Shah, Iain M Carey, Stephen DeWilde and Derek G Cook
}

\begin{abstract}
In a national primary care database sample of older people ( $\geq 65$ years), $81 \%$ ( $83588 / 103821$ ) of community and $58 \%(1702 / 2940)$ of care home residents with diabetes or heart disease had depression case finding recently recorded; $66 \%$ $(1418 / 2145)$ of community and $22 \%(26 / 118)$ of care home residents with a new depression episode had a depression-severity assessment recorded. Age, sex, and higher care home dementia prevalence did not explain these differences. Case finding and assessment of depression need to be improved in older people, particularly care home residents.

\section{Keywords}

depression; homes for the aged; elderly; quality indicators, health care.
\end{abstract}

T Harris, MSc, MD, MRCPG, GP and senior lecturer in primary care; SM Shah, MSc, FFPH, senior lecturer in public health; IM Carey, MSc, PhD, research fellow; S DeWilde, MD, MRCGP, GP and clinical research fellow; DG Cook, MSc, PhD, professor of epidemiology, Division of Population Health Sciences and Education, St George's University of London, London.

Address for correspondence

Dr Tess Harris, Division of Population Health Sciences and Education, St George's University of London, Cranmer Terrace, Tooting, London, SW17 ORE. E-mail: tharris@sgul.ac.uk

Submitted: 7 May 2010; Editor's response: 15 June 2010; final acceptance: 5 July 2010.

@British Journal of General Practice 2011; 61: 135-138.

DOI: 10.3399/bjgp11X556281

\section{INTRODUCTION}

Depression is common and often under-recognised in older people, particularly care home residents (living in nursing and residential homes), and is associated with chronic physical health problems. ${ }^{1}$ The importance of detecting depression in adults with physical health problems has been recognised by the UK Quality and Outcomes Framework (QOF), which rewards GPs for depression case finding (using two simple questions) in patients with diabetes or ischaemic heart disease. ${ }^{2}$ Case finding for depression was recorded in $88 \%$ of all such cases across UK practices in 2008-2009. ${ }^{3}$ However, no information is available on older people generally, or on those living in care homes.

Antidepressant medication and psychological therapies both have strong evidence for efficacy in older people. ${ }^{4}$ They are recommended in combination for moderate or severe depression for adults with and without chronic physical health problems, ${ }^{5,6}$ older adults, ${ }^{7}$ and care home residents. ${ }^{8}$ Antidepressant drugs are not recommended as a first-line treatment for mild to moderate depression, where psychological therapies are indicated initially. ${ }^{5,6}$

To ensure appropriate antidepressant targeting, depression severity should therefore first be assessed using validated scales. ${ }^{5-8}$ The QOF provides incentives for measurement of depression severity at treatment outset, using scales validated in primary care. $^{2}$ The three recommended measures have all been successfully used with older primary care patients: the nine-item Patient Health Questionnaire (PHQ-9); the depression subscale of the Hospital Anxiety and Depression Scale (HADS-D); ${ }^{10}$ and the Beck Depression Inventory (BDI-II). ${ }^{11}$ The HADS-D and a two-item $\mathrm{PHQ}$ have also been used successfully in care homes, ${ }^{12,13}$ but none of these scales is appropriate in dementia, where specialised scales are recommended. ${ }^{14}$

The objective of this study was to examine the use of case finding for depression and assessment of depression severity in older community and care home patients. 


\section{How this fits in}

GPs are rewarded for case finding for depression in patients with diabetes or ischaemic heart disease and for assessing severity in all new cases of depression. However, little is known about how these indicators perform in older people. This paper shows that older people in general, and those in care homes in particular, are not being screened for depression or having their depression severity assessed as often as younger adults. Differences between older community and care home samples were not explained by age, sex, or dementia prevalence and could be a manifestation of the inverse care law for care home residents.

\section{METHOD}

Data were from used from The Health Improvement Network (THIN) primary care database. A total of 413646 patients aged $\geq 65$ years were identified, from 326 English and Welsh practices, who were currently registered on the last day data were provided by the practice: most practices provided data up to February 2009; only practices providing data at least to March 2008 were included. Anonymised patient postcode linkage identified patients living in postcodes that included a nursing or residential home. Care home residents were identified by either a Read Code or multiple markers of care home residence (postcode linkage, household size, consultation location). ${ }^{15} \mathrm{~A}$ total of 403259 community and 10387 care home residents aged 65-104 years were included.
The main outcome measures were based on the QOF indicators for: (i) depression case finding in patients with diabetes or ischaemic heart disease; and (ii) assessment of depression severity in patients with a new depression episode (see Tables 1 and 2 for full definitions). QOF Read Codes were used in both cases, ${ }^{2}$ but no account was taken of exceptions recorded by GPs, as these may bias comparisons between community and care home samples. Exclusions were also applied consistently to both numerator and denominator when calculating achievement of the standards. In addition, 'QOF-equivalent' figures were calculated (Table 1) to allow direct comparison with national QOF findings.

Direct standardisation to the combined care home population in 5-year age and sex bands was used to compare community with care home rates. Confidence intervals (Cls) were calculated taking account of practice clustering, using a robust cluster variance estimate.

\section{RESULTS}

Depression screening was recorded in the last 15 months for $80.5 \%(95 \% \mathrm{Cl}=79.3$ to $81.8 \%)$ of community and $57.9 \%(95 \% \mathrm{Cl}=54.0$ to $61.8 \%)$ of care home residents with diabetes or ischaemic heart disease. Increasing age was associated with decreased screening in both settings. After exclusion of patients with dementia, the age- and sex-

\begin{tabular}{|c|c|c|c|c|c|c|}
\hline & \multicolumn{3}{|c|}{ Community $(n=403259)$} & \multicolumn{3}{|c|}{ Care homes $(n=10387)$} \\
\hline & $n$ & $\%$ & $95 \% \mathrm{Cl}$ & $n$ & $\%$ & $95 \% \mathrm{Cl}$ \\
\hline $\begin{array}{l}\text { Patients with ischaemic heart disease or diabetes and } \\
\text { no recent depression }\end{array}$ & 103821 & 100 & - & 2940 & 100 & - \\
\hline Number with case finding for depression in last 15 months ${ }^{b}$ & 83588 & 80.5 & 79.3 to 81.8 & 1702 & 57.9 & 54.0 to 61.8 \\
\hline$\%$ standardised to overall care home population ${ }^{c, d}$ & - & 73.7 & 71.9 to 75.4 & - & 57.5 & 53.6 to 61.4 \\
\hline Patients as above ${ }^{a}$ but no dementia ${ }^{e}$ ever & 101814 & 100 & - & 1795 & 100 & - \\
\hline Number with case finding for depression in last 15 months $^{\mathrm{b}}$ & 82137 & 80.7 & 79.3 to 81.9 & 1101 & 61.3 & 57.2 to 65.4 \\
\hline$\%$ standardised to overall care home population ${ }^{\mathrm{c}, \mathrm{d}}$ & - & 74.0 & 72.3 to 75.8 & - & 61.1 & 57.0 to 65.1 \\
\hline \multicolumn{7}{|l|}{ Sex and age } \\
\hline Females aged $65-74$ years & 19106 & 81.0 & 79.7 to 82.3 & 77 & 75.3 & 65.3 to 85.3 \\
\hline Females aged $75-84$ years & 19162 & 79.6 & 78.3 to 81.0 & 355 & 62.8 & 57.0 to 68.6 \\
\hline Females aged $85-104$ years & 7691 & 71.1 & 69.0 to 73.2 & 827 & 56.8 & 51.8 to 61.9 \\
\hline Males aged $65-74$ years & 29041 & 82.5 & 81.3 to 83.8 & 98 & 69.4 & 60.3 to 78.4 \\
\hline Males aged $75-84$ years & 21473 & 82.9 & 81.5 to 84.2 & 227 & 65.6 & 59.0 to 72.3 \\
\hline Males aged $85-104$ years & 5341 & 78.1 & 76.2 to 79.9 & 211 & 63.0 & 56.1 to 70.0 \\
\hline Patients based on QOF-equivalent calculation ${ }^{\dagger}$ & 104593 & 100 & - & 2680 & 100 & - \\
\hline Number with case finding for depression in last 15 months ${ }^{b}$ & 85198 & 81.5 & 80.2 to 82.7 & 1736 & 64.8 & 61.1 to 68.4 \\
\hline$\%$ standardised to overall care home population ${ }^{c, d}$ & - & 75.2 & 73.6 to 76.9 & - & 64.3 & 60.7 to 68.0 \\
\hline
\end{tabular}

${ }^{a}$ Patients registered for at least 90 days, with a code of diabetes or ischaemic heart disease ever, at least 90 days from the end of follow-up and no new depression episode in the last 15 months. ${ }^{b}$ Case finding for depression based on two standard screening questions being recorded. ${ }^{\circ}$ Standardisation was for age in 5-year bands and sex using direct standardisation to the care home population; $95 \% \mathrm{Cls}$ allow for clustering by practice. ${ }^{\mathrm{d}}$ All standardised community and care home differences are significant at $\mathrm{P}<0.001$. ${ }^{\mathrm{D}}$ Dementia diagnosis was based on Read Codes used in the QOF, ${ }^{2}$ with additional codes for cognitive decline. ${ }^{\dagger} Q O F$ calculation differs from ${ }^{\text {a }}$ by including patients with recent case finding for depression in the denominator regardless of exclusion criteria. QOF = Quality and Outcomes Framework. 


\begin{tabular}{|c|c|c|c|c|c|c|}
\hline & \multicolumn{3}{|c|}{ Community $(n=403259)$} & \multicolumn{3}{|c|}{ Care homes $(n=10387)$} \\
\hline & $n$ & $\%$ & $95 \% \mathrm{Cl}$ & $n$ & $\%$ & $95 \% \mathrm{Cl}$ \\
\hline Patients with new episode of depression ${ }^{a}$ & 2145 & 100 & - & 118 & 100 & - \\
\hline Number with assessment of severity within 28 days & 1418 & 66.1 & 62.9 to 69.3 & 26 & 22.0 & 14.0 to 30.1 \\
\hline$\%$ standardised to overall care home population ${ }^{\mathrm{c}, \mathrm{d}}$ & - & 55.0 & 47.8 to 62.2 & & 22.6 & 15.1 to 30.2 \\
\hline Patients as above ${ }^{a}$ with no dementia ${ }^{e}$ ever & 2076 & 100 & - & 79 & 100 & \\
\hline Number with assessment of severity within 28 days $^{b}$ & 1389 & 66.9 & 63.7 to 70.2 & 20 & 25.3 & 15.4 to 35.2 \\
\hline$\%$ standardised to overall care home population ${ }^{\mathrm{c}, \mathrm{d}}$ & - & 56.3 & 49.1 to 63.5 & & 26.5 & 17.0 to 36.0 \\
\hline Patients based on QOF-equivalent calculation ${ }^{\dagger}$ & 2617 & 100 & - & 121 & 100 & \\
\hline Number with assessment of severity within 28 days ${ }^{b}$ & 1922 & 73.4 & 70.6 to 76.3 & 36 & 29.8 & 21.1 to 38.4 \\
\hline$\%$ standardised to overall care home population ${ }^{c, d}$ & - & 63.2 & 56.2 to 70.1 & - & 30.1 & 22.0 to 38.1 \\
\hline
\end{tabular}

${ }^{\text {a }}$ Patients registered for at least 90 days with a new diagnosis of depression in period 91-450 days from end of follow-up and no depression severity assessment $>365$ days before end of follow-up. ${ }^{b}$ Depression severity assessment using an assessment tool validated for use in primary care. ${ }^{\mathrm{c} S t a n d a r d i s a t i o n ~ w a s ~ f o r ~ a g e ~ i n ~}$ 5 -year bands and sex using direct standardisation to the care home population, 95\% Cls allow for clustering by practice. ${ }^{\mathrm{d}} \mathrm{All}$ standardised community and care home differences are significant at $\mathrm{P}<0.001$. ${ }^{\mathrm{e}}$ Dementia diagnosis was based on Read Codes used in $Q O F^{2}$ with additional codes for cognitive decline. ${ }^{\dagger} \mathrm{QOF}$ calculation differs from ${ }^{a}$ by including patients with depression severity assessment within 28 days in denominator regardless of exclusion criteria.

standardised screening rates were $74.0 \%$ in the community and $61.1 \%$ in care homes (Table 1 ).

An assessment of depression severity was recorded in $66.1 \%(95 \% \mathrm{Cl}=62.9$ to $69.3 \%)$ of community and $22.0 \%(95 \% \mathrm{Cl}=14.0$ to $30.1 \%)$ of care home residents with a new depression episode. This difference in rates persisted after exclusion of patients with dementia and age and sex adjustment (56.3\% in community and $26.5 \%$ in care homes; Table 2).

\section{DISCUSSION}

\section{Summary of main findings}

Older care home patients with diabetes or ischaemic heart disease are less likely to have case finding for depression than community patients, and the oldest age groups are less likely to be screened in both settings. Care home patients with new depression episodes are less likely to have a depression-severity measure recorded than community patients. The differences in performance of these QOF depression indicators between community and care homes are not explained by age and sex differences or by the greater dementia prevalence in care homes. Given the higher risk of depression in care homes, the differences in performance of these QOF depression indicators may be a manifestation of inverse care for care home residents.

\section{Strengths and limitations of the study}

This is the first nationally representative study to examine the performance of the QOF depression indicators in older people. The novel methodology allows separate identification of care home residents, which has not previously been possible in large UK samples. Measurement of the first indicator (depression case finding in patients with diabetes or ischaemic heart disease) is potentially biased by shorter registration times for care home patients, because of rapid patient turnover; but limiting the analysis to patients registered for 15 months in both settings made very little difference to the results (not shown). A potential weakness of the data is reliance on the quality of routine recording, but the variables used are likely to be well recorded, as these definitions are used to determine practice payments.

\section{Comparison with existing literature}

The finding in this study of a QOF-equivalent estimate of $82 \%$ for depression case finding for older people with diabetes or heart disease in the community was lower than the value of $88 \%$ recorded for all-age adults across UK practices in 2007-2008 and 2008-2009. ${ }^{3}$ Combined with the finding that screening likelihood decreased with increasing age for both samples, this suggests that for GPs, age is an important barrier to using the screening tests.

Similarly, the study's QOF-equivalent estimate of $73 \%$ for assessment of depression severity for older people in the community was lower than the published attainment of $91 \%$ and $92 \%$ of adults in 2007-2008 and 2008-2009 respectively. ${ }^{3}$ This suggests that depression severity is less frequently recorded in older people, despite these measures having been successfully used with older people in primary care..$^{9-11}$

Kendrick et al demonstrated that even when depression severity is recorded in older people, these patients are less likely to be started on antidepressant medication or referred..$^{16}$ However, the findings of the present study worryingly suggest that older people are even less likely to receive appropriate 
management when diagnosed with depression.

The severity-assessment scales recommended by the QOF are not appropriate in dementia, but even after excluding patients with dementia, there was evidence of their use in only $25 \%$ of care home patients.

Possible reasons for their low use in care homes include the following: practical difficulties in accessing the instruments or in remembering to use them when out of the surgery; concerns about possible undiagnosed dementia or the presence of other diseases making assessment difficult (for example, deafness, dysphasia); and a belief that severity assessment may not affect treatment (for example, if alternatives to medication are difficult to access for this population). Enabling access to computerised records with clinical prompts in care homes could address at least some of the practical barriers. Alternatively, there is evidence that GPs are sceptical about the value of depression-severityassessment scores compared to clinical judgement, ${ }^{17}$ and this may lead to their omission in patients for whom administration may be perceived as difficult or where psychological therapies are not accessible, such as care home residents.

\section{Implications for clinical practice}

The study findings suggest that older people in general, and those in care homes in particular, are not being screened for depression in the presence of diabetes or ischaemic heart disease or having their depression severity assessed as often as younger adults. GPs need to consider improving their attainment of these depression indicators in older people, particularly for those living in care homes, as they are at markedly increased risk of depression.

\section{Funding body}

The study was funded by a grant from the BUPA Foundation, an independent medical research charity. The funder had no role in the design, execution, analysis, interpretation, or writing of this paper.

\section{Ethical approval}

The study was approved by the South-East Multicentre Research Ethics Committee (study reference: 08/H1102/33).

\section{Competing interests}

The authors have stated that there are none.

\section{Acknowledgements}

Everyone who has contributed significantly to the work is listed as an author.

\section{Discuss this article}

Contribute and read comments about this article on the

Discussion Forum: http://www.rcgp.org.uk/bjgp-discuss

\section{REFERENCES}

1. Djernes JK. Prevalence and predictors of depression in population of elderly: a review. Acta Psychiatr Scand 2006; 113(5): 372-387.

2. Department of Health. New GMS Contract QOF implementation. Dataset and business rules. London: Department of Health, 2006.

3. NHS Information Centre. Quality and Outcomes Framework database. Leeds: NHS Information Centre, 2009.

4. Frederick JT, Steinman LE, Prohaska T, et al. Community-based treatment of late life depression an expert panel-informed literature review. Am J Prev Med 2007; 33(3): 222-249.

5. National Institute for Health and Clinical Excellence. Depression: the treatment and management of depression in adults (update). Clinical Guideline 90. London: National Institute for Health and Clinical Excellence, 2009.

6. National Institute for Health and Clinical Excellence. Depression in adults with a chronic physical health problem: treatment and management. Clinical Guideline 91. London: National Institute for Health and Clinical Excellence, 2009.

7. Steinman LE, Frederick JT, Prohaska T, et al. Recommendations for treating depression in community-based older adults. Am J Prev Med 2007; 33(3): 175-181.

8. Long Term Care Professional Leadership Council, American College of Health Care Administrators, American Medical Directors Association, American Society of Consultant Pharmacists, National Association Directors of Nursing Administration/Long Term Care. Use of antidepressants in nursing home residents. A joint statement of the members of the Long Term Care Professional Leadership Council (LTCPLC). Consult Pharm 2008; 23(3): 231-234.

9. Weinberger MI, Mateo C, Sirey JA. Perceived barriers to mental health care and goal setting among depressed, community-dwelling older adults. Patient Prefer Adherence 2009; 3: 145-149.

10. Watts SC, Bhutani GE, Stout IH, et al. Mental health in older adult recipients of primary care services: is depression the key issue? Identification, treatment and the general practitioner. Int J Geriatr Psychiatry 2002; 17(5): 427-437.

11. Serfaty MA, Haworth D, Blanchard M, et al. Clinical effectiveness of individual cognitive behavioral therapy for depressed older people in primary care: a randomized controlled trial. Arch Gen Psychiatry 2009; 66(12): 1332-1340.

12. Watson LC, Zimmerman S, Cohen LW, Dominik R. Practical depression screening in residential care/assisted living: five methods compared with gold standard diagnoses. Am J Geriatr Psychiatry 2009; 17(7): 556-564.

13. Sackley CM, Hoppitt TJ, Cardoso K. An investigation into the utility of the Stroke Aphasic Depression Questionnaire (SADQ) in care home settings. Clin Rehabil 2006; 20(7): 598-602.

14. Alexopoulos GS, Abrams RC, Young RC, Shamoian CA. Cornell Scale for Depression in Dementia. Biol Psychiatry 1988; 23(3): 271-284.

15. Shah SM, Carey IM, Harris T, et al. Identifying the clinical characteristics of older people living in care homes using a novel approach in a primary care database. Age Ageing 2010; 39(5): 617-623.

16. Kendrick T, Dowrick C, McBride A, et al. Management of depression in UK general practice in relation to scores on depression severity questionnaires: analysis of medical record data. BMJ 2009; 338: b750.

17. Dowrick C, Leydon GM, McBride A, et al. Patients' and doctors' views on depression severity questionnaires incentivised in UK quality and outcomes framework: qualitative study. BMJ 2009; 338: b663. 